\title{
Supersonic Flows near Blunted Cylinders, Giving off Opposite Jets: CFD Search for Self-Oscillatory Regimes
}

\author{
V. I. Pinchukov \\ In-te of Computational Technologies, \\ Siberian division of Russian Academy of Sc., \\ Novosibirsk, Russia
}

\begin{abstract}
Parametric numerical studies of supersonic flows near spherically blunted cylinders, giving off opposite supersonic jets from forehead surfaces, are carried out. Two-dimensional Euler equations of a polytropic gas with the specific heats ratio 1.4 are applied. An explicit Godunov second order scheme is used. Self-oscillatory flows are found for free stream Mach numbers $1.1 \leq M_{\infty} \leq 2.3$. It is shown, that flows are steady for sufficiently large jet half-angles, jets are destroying and a process of this destroying produces self-oscillations for middle jet half-angles, jets propagate at a greater distance from spherical blunts of cylinders for sufficiently small jet half-angles. This paper is devoted to investigations of unsteady flows mainly for middle jet half-angles.
\end{abstract}

\section{Keywords - Euler equations, CFD studies, self-oscillations, compressible flows}

\section{INTRODUCTION}

This paper is devoted to continuation of a search for unsteady compressible flows, started in [1]. Flow self-oscillations are supposed to be resulted from resonance interactions of flow "active" elements, namely, elements, which amplify disturbances. The hypothesis [1] is used that contact discontinuities and intersection points (lines in $3 \mathrm{~d}$ case) of shocks with shocks or shocks with contact discontinuities compose the flow set of "active" elements. A search for new unsteady flows is conducted by investigations of flows, containing the most number of "active" elements.

Opposite jets, out-flowing from forehead surfaces of blunted bodies, may be used for protecting bodies from heating by a mainstream (see, for example, [2]-[4]). Self-oscillatory regimes are not observed in previous investigations. At the same time calculated here flow fields contain three shock waves, two contact discontinuities and some intersection points [1]. So these flows may be supposed to produce self-oscillations, according to the formulated above hypothesis. It seems that there is contradiction between present results and other investigations. To settle this contradiction it should be noted that, first, flows near blunted bodies with opposite jets are defined by large number of control parameters and depend on body and nozzle forms, consequently, require more thorough investigations, second, subsonic or transonic jets are usually used for cooling of the bodies while present considerations deal with jet Mach numbers $M_{j e t} \geq 2.5$.

Numerical studies of supersonic flows near blunted cylinders, giving of opposite jets, started in [1], where existence of unsteady regimes was noted. Self-oscillatory regime of flows near blunted cylinders with opposite jets were studied in [5],[6] at free stream Mach numbers, closed to 1 . Here these investigations are continued.

A class of jet flows with self-sustained oscillations contains three families. Besides present new family, there are two known families, containing: 1. Flows near supersonic jets, inflowing to forward facing cavities (see, for example, [7]-[10]); 2. Flows near supersonic jets, impinging on plates [11]-[19].

\section{CFD DESIGN APPROACH}

A computational domain and a mesh may be seen in figure 1. The blunted cylinder wall is shown by a bold line. Opposite supersonic jets outflow from nozzles in forehead parts of blunted cylinders. These jets are supposed to be spherically symmetrical. Jet velocities at exits of nozzles are normal to spherical surfaces. 


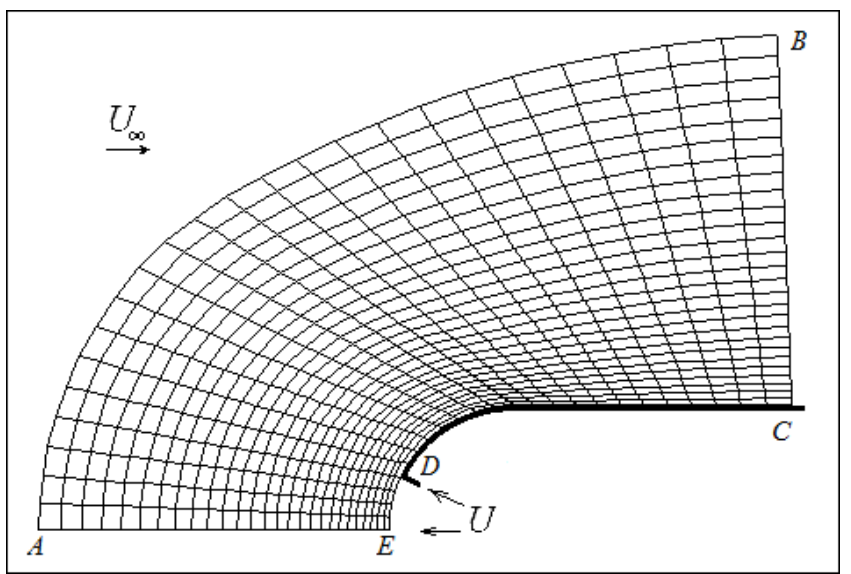

Fig. 1. Mesh and computational domain schematic representation.

Boundary conditions for computations are zero value of the normal velocity and extrapolation relations for all other variables along the body surface (boundary $D C$ ), extrapolations on the outflow boundary $B C$ at right side of numerical domain (see fig 1), prescribed variables on the inflow forehead boundary $\mathrm{AB}$ and on the spherical boundary $D E$, corresponding to the opposite jet, zero value of the radial velocity and extrapolations at symmetry axis $A E$.

The second order two step version of Godunov conservative method [20] with approximate linear solution of Riman problem is employed here. Algorithms of slopes limitation of left and right extrapolation curves are used to damp false oscillations near discontinuities. Review of such algorithms of damping false oscillations are presented in [21]. Navier-Stokes viscous terms are included to Euler equations and the Smagorinsky artificial viscosity [22] is used for additional damping of false oscillations. This viscosity is calculated by equations

$\mu=\rho|S|\left(C_{s} \Delta\right)^{2},|S|=\left(2 S_{i k} S_{i k}\right)^{1 / 2}$,

$\mathrm{S}_{i k}=\left(\partial \mathrm{u}_{i} / \partial \mathrm{x}_{k}+\partial \mathrm{u}_{k} / \partial \mathrm{x}_{i}\right) / 2, \Delta=\Delta \xi \Delta \eta\left(\mathrm{x}_{\zeta} \mathrm{y}_{\eta}-\mathrm{y}_{\zeta} \mathrm{x}_{\eta}\right) /\left(\operatorname{Min}\left(\Delta \xi^{2}\left(\mathrm{x}_{\zeta}^{2}+\mathrm{y}_{\zeta}^{2}\right), \Delta \eta \eta_{\eta}^{2}\left(\mathrm{x}_{\eta}^{2}+\mathrm{y}_{\eta}^{2}\right)\right)\right)$

where functions $x=x(\xi, \eta), y=y(\xi, \eta)$ perform mapping of the unit square $\{0 \leq \xi \leq 1,0 \leq \eta \leq 1\}$ to a curvilinear quadrangle on the plane of physical variables, $\Delta \xi=1 / N_{\zeta}, \Delta \eta=1 / N_{\eta}, N_{\zeta}, N_{\eta}$ - numbers of intervals of the quadrangular mesh in a unit square, $C_{s}$ - constant, which is chosen in trial computations, $C_{s}=0.85$. The $490 \times 544$ mesh is used.

Numerical calculations deal with dimensionless variables. These variables are defined as relations of initial variables and next parameters of the free stream flow or radiuses of cylinders $r: p_{\infty}$ - for pressure, $\rho_{\infty}$ - for density, $\sqrt{p_{\infty} / \rho_{\infty}}-$ for velocity, $r-$ for space variables, $r / \sqrt{p_{\infty} / \rho_{\infty}}$ - for time.

The intensity of flow oscillations may be measured by sound pressure level at some point: $S P L=10 \log 10\left(\overline{p^{2}} / p_{r e f}^{2}\right), \overline{p^{2}}=\sum_{n}\left(p_{n}-\bar{p}\right)^{2} / N, p_{r e f}=20 \mathrm{mkPa} / p_{\infty}$,

where $p_{\infty}=101325 \mathrm{~Pa}$ (the air pressure under normal conditions) is used since dimensionless variables are dealt here. Sound pressure levels at the intersection point of spherical and cylindrical parts are represented in three tables below. 


\section{FREE STREAM MACH NUMBERS $1.1 \leq M_{\infty} \leq 1.3$.}

Main purpose of this paper is to find unsteady compressible flows near blunted cylinders, giving off supersonic opposite jets, at free stream Mach numbers, exceeding Mach numbers, considered in (Pinchukov, 2014, 2016, 2018). Studied here flows are defined by five control parameters: $M_{\infty}, M_{j e t}, \rho_{j e t}, p_{j e t}, \sigma_{j e t}$ - free stream and jet Mach numbers, jet density, jet pressure, a jet half-angle. Large number of control parameters makes systematic parametric study of these flows too expensive. A search for new self-oscillatory flows is performed by moving step by step in the greater Mach number direction from any initial flows, for example, published in [5], [6]. Results of a search are represented in table 1-3. The first column of these tables contains numbers of variants, columns 2-6 show control parameters of calculated flows, the last column shows results of computations, namely, SPL data at the intersection point of spherical and cylindrical parts of considered bodies.

Table 1

Sound pressure levels at $1.1 \leq M_{\infty} \leq 1.3$.

\begin{tabular}{lllllll}
\hline $\mathrm{N}$ & $M_{\infty}$ & $M_{j e t}$ & $P_{j e t}$ & $\boldsymbol{\rho}_{j e t}$ & $\operatorname{Sin}\left(\sigma_{j e t}\right)$ & $S P L, d b$ \\
\hline 1 & 1.1 & 3.0 & 0.5 & 0.583 & $12 / 30$ & 174.8 \\
\hline 2 & 1.1 & 3.5 & 0.4 & 0.625 & $1 / 3$ & 172.3 \\
\hline 3 & 1.1 & 3.5 & 0.4 & 0.223 & $11 / 30$ & 165.1 \\
\hline 4 & 1.2 & 3.0 & 0.5 & 0.340 & $1 / 3$ & 165.4 \\
\hline 5 & 1.2 & 3.0 & 0.3 & 0.567 & $11 / 30$ & 163.0 \\
\hline 6 & 1.2 & 4.0 & 0.292 & 0.469 & $1 / 3$ & 169.6 \\
\hline 7 & 1.3 & 3.0 & 0.502 & 0.484 & $1 / 3$ & 164.4 \\
\hline 8 & 1.3 & 4.5 & 0.233 & 0.465 & $13 / 30$ & 162.2 \\
\hline 9 & 1.3 & 4.5 & 0.233 & 0.465 & $12 / 30$ & 170.0 \\
\hline 10 & 1.3 & 4.5 & 0.233 & 0.465 & $11 / 30$ & 169.8 \\
\hline 11 & 1.3 & 4.5 & 0.233 & 0.465 & $1 / 3$ & 167.5 \\
\hline 12 & 1.3 & 4.5 & 0.233 & 0.465 & $9 / 30$ & - \\
\hline 13 & 1.3 & 4.5 & 0.233 & 0.465 & $8 / 30$ & \\
\hline
\end{tabular}

It is interesting to consider variants 8-13 in this table. Control parameters $M_{\infty}, M_{j e t}, \rho_{j e t}, p_{j e t}$, are identical for these variants, the parameter $\sigma_{j e t}$ is varied from $\arcsin (13 / 30)$ to $\arcsin (8 / 30)$. It may be seen (column 7 of table 1), that oscillations of the variant 8 with the largest parameter $\sigma_{j e t}$ are weak. If to increase the parameter $\sigma_{j e t}$, then the steady state regime is observed. Figure 2 a shows the density distribution in the flow number 8.

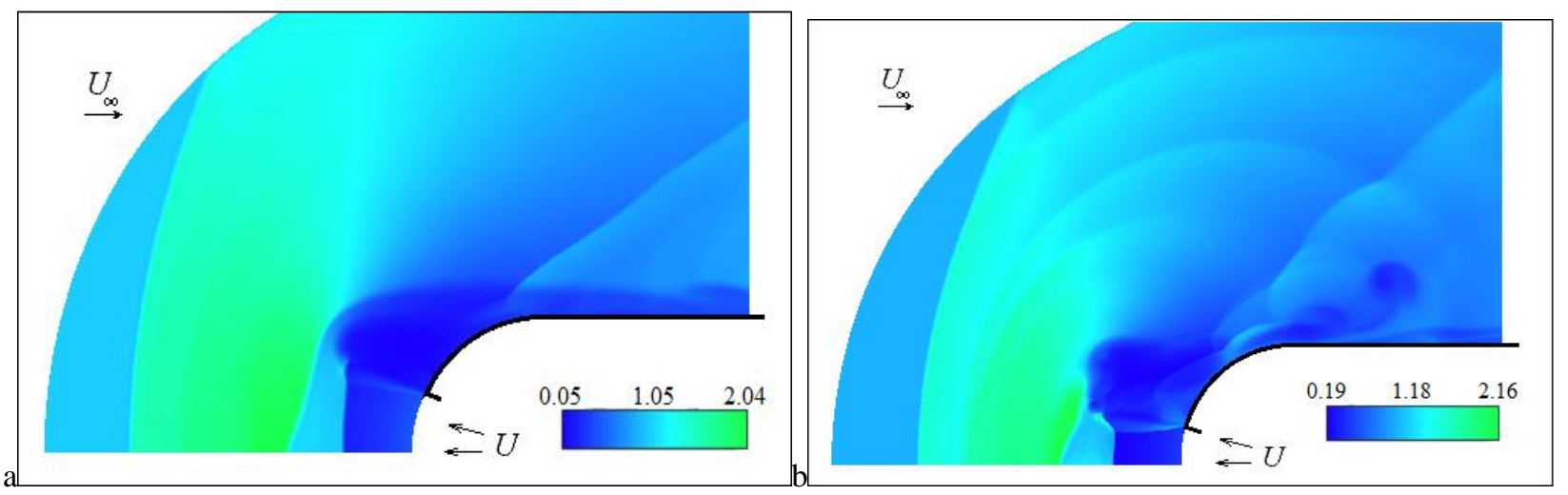

Figs. 2. Density distributions, $\mathrm{a}-\sigma_{j e t}=\arcsin (13 / 30), \mathrm{b}-\sigma_{j e t}=\arcsin (1 / 3)$. 
Decreasing of the parameter $\sigma_{j e t}$ results appearing of more intensive oscillations. Figure $2 \mathrm{~b}$ shows the density distribution in the flow number 11. Intensive oscillations near the bold part of the cylinder produce shock waves, moving towards the main shock wave, and vortexes, drifting above cylinder surface.

Next decreasing of the parameter $\sigma_{j e t}$ changes the flow regime. If variants 8-12 correspond to destroying of out-flowing jet, and a process of jet destroying provides generation of fluctuations, the variant 13 corresponds to propagation of the jet towards the inflow boundary. Figure 3 shows the density distribution in the flow number 13. The flow regime is not defined at this time instant. If to continue modeling of this flow, the main shock wave gets the inflow boundary $A B$. Next calculations of this flow become unable. Present CFD approach is not applicable to calculations of such flows.

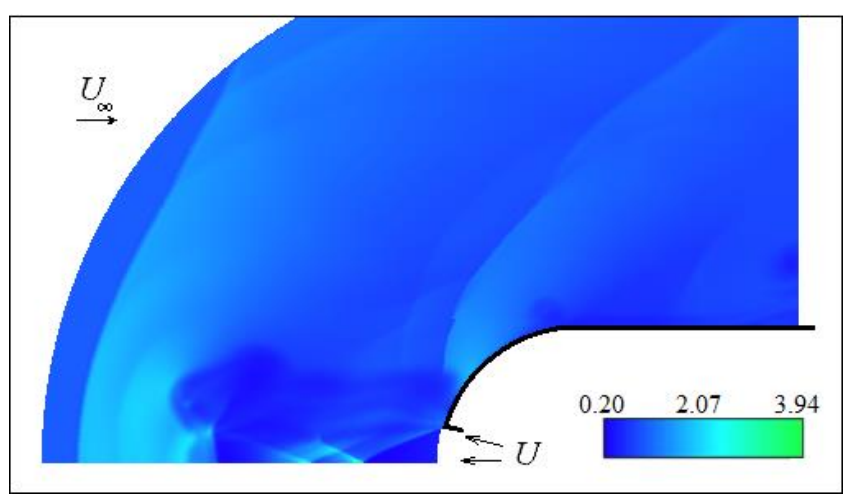

Fig. 3. The density distribution, $\sigma_{j e t}=\arcsin (8 / 30)$.

Similar dependence of flow regimes on a jet half-angle $\sigma_{\text {jet }}$ is observed for all considered here free stream Mach numbers. But flows with new regime (like the flow 13) and flows with weak oscillations (like the flow 8) are omitted for other free stream Mach numbers.

\section{FREE STREAM MACH NUMBERS $1.4 \leqslant M_{\infty} \leqslant 1.8$.}

Results of a CFD search for middle Mach numbers are represented in table 2.

Table 2

Sound pressure levels at $1.4 \leq M_{\infty} \leq 1.8$.

\begin{tabular}{lllllll}
\hline $\mathrm{N}$ & $M_{\infty}$ & $M_{\text {jet }}$ & $P_{j e t}$ & $\boldsymbol{\rho}_{\text {jet }}$ & $\operatorname{Sin}\left(\sigma_{j e t}\right)$ & $S P L, d b$ \\
\hline 1 & 1.4 & 4.5 & 0.233 & 0.465 & $12 / 30$ & 161.7 \\
\hline 2 & 1.4 & 5.5 & 0.157 & 0.459 & $1 / 3$ & 168.3 \\
\hline 3 & 1.4 & 5.5 & 0.394 & 0.289 & $1 / 3$ & 170.0 \\
\hline 4 & 1.4 & 5.5 & 0.25 & 0.347 & $1 / 3$ & 179.5 \\
\hline 5 & 1.5 & 5.5 & 0.25 & 0.289 & $1 / 3$ & 177.6 \\
\hline 6 & 1.5 & 5.5 & 0.25 & 0.347 & $1 / 3$ & 174.3 \\
\hline 7 & 1.6 & 5.5 & 0.25 & 0.289 & $1 / 3$ & 176.3 \\
\hline 8 & 1.6 & 5.5 & 0.2 & 0.289 & $12 / 30$ & 164.0 \\
\hline 9 & 1.7 & 5.5 & 0.25 & 0.289 & $1 / 3$ & 179.2 \\
\hline 10 & 1.7 & 5.5 & 0.25 & 0.347 & $1 / 3$ & 173.53 \\
\hline 11 & 1.7 & 5.5 & 0.25 & 0.347 & $12 / 30$ & 151.9 \\
\hline 12 & 1.8 & 5.5 & 0.25 & 0.289 & $1 / 3$ & 174.2 \\
\hline 13 & 1.8 & 5.5 & 0.25 & 0.347 & $1 / 3$ & \\
\hline
\end{tabular}

Figure 4a shows the density distribution for the unsteady flow 6 in table 2. Intensive self-oscillations produce shock waves, moving towards the main bold shock wave, and vortexes, drifting above the cylinder surface. 

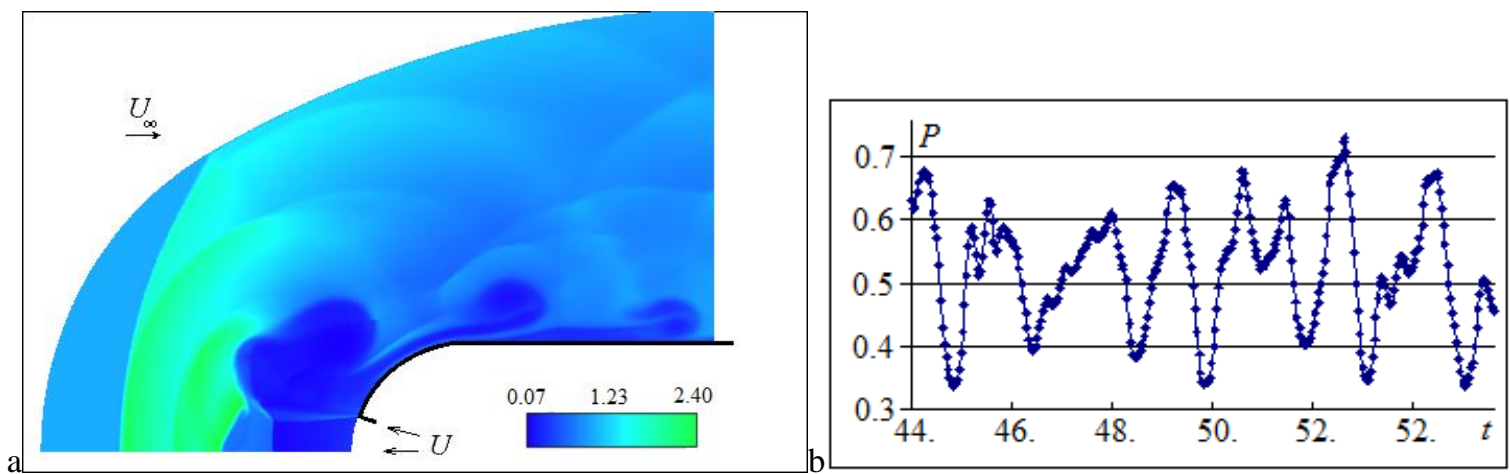

Figs. $4 . M_{\infty}=1.5, \mathrm{a}-$ the density distribution, $\mathrm{b}$ - the pressure history.

The pressure history is shown in figure $4 \mathrm{a}$ for this flow. Seven points of minimal values of pressure may be seen in this figure. So main tone period of this flow may be evaluated approximately by the formulae $\tau=\left(\mathrm{t}_{7}-\mathrm{t}_{1}\right) / 6=1.68$.

V. Free Stream Mach Numbers $1.9 \leq M_{\infty} \leq 2.3$.

Examples of numerically found self-oscillatory flows are shown in table 3.

Table 3

Sound pressure levels at $1.9 \leq M_{\infty} \leq 2.5$.

\begin{tabular}{lllllll}
\hline $\mathrm{N}$ & $M_{\infty}$ & $M_{j e t}$ & $p_{j e t}$ & $\rho_{j e t}$ & $\operatorname{Sin}\left(\sigma_{j e t}\right)$ & $S P L, d b$ \\
\hline 1 & 1.9 & 5.5 & 0.25 & 0.289 & $1 / 3$ & 170.0 \\
\hline 2 & 1.9 & 5.5 & 0.25 & 0.405 & $1 / 3$ & 169.0 \\
\hline 3 & 2.0 & 4.5 & 0.50 & 0.432 & $1 / 3$ & 169.1 \\
\hline 4 & 2.0 & 5.5 & 0.25 & 0.405 & $1 / 3$ & 163.3 \\
\hline 5 & 2.0 & 5.5 & 0.35 & 0.289 & $1 / 3$ & 171.7 \\
\hline 6 & 2.15 & 4.5 & 0.5 & 0.432 & $1 / 3$ & 166.9 \\
\hline 7 & 2.15 & 4.5 & 0.5 & 0.432 & $9 / 30$ & 171.2 \\
\hline 8 & 2.15 & 4.5 & 0.5 & 0.432 & $8 / 30$ & 166.3 \\
\hline 9 & 2.3 & 4.5 & 0.5 & 0.289 & $9 / 30$ & 169.5 \\
\hline 10 & 2.3 & 5.5 & 0.5 & 0.289 & $9 / 30$ & 175.6 \\
\hline 11 & 2.3 & 5.5 & 0.5 & 0.434 & $12 / 30$ & 172.8 \\
\hline 12 & 2.3 & 4.5 & 0.5 & 0.648 & $1 / 3$ & 177.9 \\
\hline 13 & 2.3 & 5.5 & 0.5 & 0.434 & $9 / 30$ & 172.4 \\
\hline 14 & 2.3 & 5.5 & 0.5 & 0.434 & $8 / 30$ & \\
\hline
\end{tabular}

Figure 5a shows the density distribution for the flow 11 in table 3. This figure shows shock waves moving from the body bold region to the main forehead shock wave, and vortexes, appearing near the body bold and moving downstream along the cylinder. 


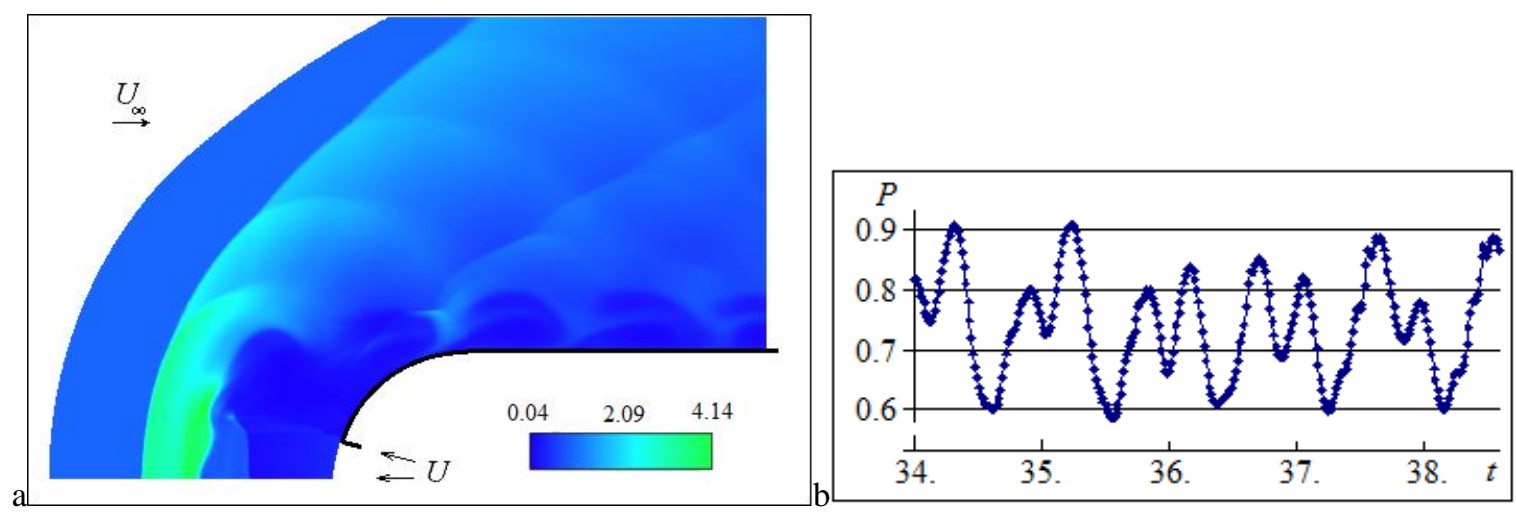

Figs. 5. $M_{\infty}=2.3$, a -the density distribution, $\mathrm{b}$ - the pressure history.

Figure $5 \mathrm{~b}$ shows the pressure history for this flow at the interface point of spherical and cylindrical parts of the body. Five points of pressure minimal values in this figure divide this graph on periods. So present flow may be considered very approximately as periodical with the period $\tau=\left(\mathrm{t}_{5}-\mathrm{t}_{1}\right) / 4=0.90$.

\section{CONCLUTIONS}

Interactions of supersonic streams with blunted bodies, giving off supersonic jets, are studied. Previous investigations [5]-[6] allowed to find unsteady regimes of these interactions at free stream Mach numbers, closed to 1. The Mach numbers interval $1.1 \leq M_{\infty} \leq 2.3$ of unsteady regimes is established here.

Comparison of variants 8-13 in table 1 shows, that flows are steady for sufficiently large jet half-angles, jets are destroying and a process of this destroying produces self-oscillations for middle jet half-angles, jets get greater distance from spherical blunts of cylinders for sufficiently small jet half-angles.

As a jet velocity or jet pressure or jet density decreases, a jet gets less distance from spherical blunt as a result of jet braking by a contrary free stream. A steady state regime takes place, if this distance is sufficiently small.

To define more exactly regions with any flow regimes in 5D space of control parameters, both CFD modeling and experimental studies are necessary.

\section{REFERENCES}

[1] V. I. Pinchukov, "Modeling of Self-oscillations and a Search for New Self-oscillatory Flows," Mathematical Models and Computer Simulations, 4(2), 2012, 170-178.

[2] C. H. E. Warren, “An Experimental Investigation of the Effect of Ejecting a Coolant Gas at the Nose of a Bluff Body,” J. of Fluid Mechanics, Vol.8, No. 3, 1960, 400-417.

[3] K. Hayashi, S. Aso, "Effect of Pressure Ratio on Aerodynamic Heating Reduction Due to Opposing Jet," AIAA 2003-4041, 2003.

[4] I. Tamada, S. Aso, Y. Tani, "Reducing Aerodynamic Heating by the Opposing Jet in Supersonic and Hypersonic Flows," AIAA 2010-991, 2010.

[5] V. I. Pinchukov, "Numerical Simulations of Self-oscillatory Flows near Blunted Bodies, Giving off Opposite Jets." Intern. J. of Mechanical Engineering and Applications 2(1), 2014, 5-10.

[6] V. I. Pinchukov, "Self-oscillatory Flows near Blunted Bodies, Giving off Opposite Jets: CFD Study." Intern. J. of Engineering and Innovative Technology, 6(5), 2016, 41-46.

[7] J. Hartmann, "On a New Method for the Generation of Sound Waves." Phys. Rev., 20(6), 1922, 719-726.

[8] S. Murugappan, E. Gutmark, "Parametric Study the Hartmann-Sprenger Tube." Experiments in Fluids, 38(6), 2005, 813-823.

[9] J. Kastner, M. Samimy, "Development and Characterization of Hartmann Tube Fluid Actuators for High-speed Control." American Institute of Aeronautics and Astronautics J., 40(10), 2002, 1926-1934.

[10] G. Raman, E. Envia, T. J. Bencic, “Jet Cavity Interaction Tones.” American Institute of Aeronautics and Astronautics J., 40(8), (2002), $1503-1511$.

[11] W. Wu, U. Piomelli, "Large-eddy simulation of impinging jets with embedded azimuthal vorti ces." J. of Turbulence, 16(10), 2014, 44-66.

[12] C.-Y. Kuo, A. P. Dowling, “Oscillations of a Moderately Underexpanded Choked Jet Impinging upon a Flat Plate.” J. Fluid Mech., 315, 1996, 267-291.

[13] Y. Sakakibara, J. Iwamoto, "Numerical Study of Oscillation Mechanism in Underexpanded Jet Impinging on Plate.” J. Fluids Eng., 120, 1998, 477. 
[14] B. Henderson, J. Bridges, M. Wernet, “An Experimental Study of the Oscillatory Flow Structure of Tone-producing Supersonic Impinging Jets.” J. Fluid Mech., 542, 2005, 115-137.

[15] J. Berland, C. Bogey, C. Bailly, "Numerical Study of Screech Generation in a Planar Supersonic Jet." Phys. Fluids, 19, 2007, 75-105.

[16] D. J. Bodony, S. K. Lele, "On Using Large-eddy Simulation for the Prediction of Noise from Cold and Heated Turbulent Jets." Phys. Fluids, 17, 2005.

[17] C. Bogey, C. Bailly, "Computation of a High Reynolds Number Jet and Its Radiated Noise Using Large Eddy Simulation Based on Explicit Filtering." Comput. Fluids, 35, 2006, 1344-1358.

[18] T. Cheng, K. Lee, "Numerical Simulations of Underexpanded Supersonic Jet and Free Shear Layer Using WENO Schemes." Int. J. Heat Fluid Flow, 26(5), 2005, 755-770.

[19] V. I. Pinchukov, "Numerical Modeling of Unsteady Flows with Transient Regimes." Comput. Mathem. and Mathem. Physics, 49 (10), 2009 , $1844-1852$.

[20] S. K. Godunov, "A Difference Method for Numerical Calculation of Discontinuous Solutions of the Equations of Hydrodynamics." Mat. Sb. (N.S.), 47(89):3, 1959, 271-306

[21] P. Woodward, "The Numerical Simulation of Two-Dimensional Fluid Flow with Strong Shocks Journal of Comput. Physics, 54, 1984, 115-173.

[22] J. Smagorinsky, "General Circulation Experiments with the Primitive Equations. I. The Basic Experiment.” Monthly Weather Review, 91, 1963, 99-164. 